\title{
A Case of Tacrolimus-Induced Posterior Reversible Encephalopathy Syndrome Initially Presenting as a Bilateral Optic Neuropathy
}

\author{
Sumana S. Kommana ${ }^{a} \quad$ Upneet Bains $^{a} \quad$ Vivian Fasula $^{b}$ Jeffrey Henderer ${ }^{a}$ \\ aDepartment of Ophthalmology, Temple University Hospital, Philadelphia, PA, USA; \\ ${ }^{b}$ Department of Ophthalmology, Veterans Affairs Medical Center, Bay Pines, FL, USA
}

\section{Keywords}

Tacrolimus · Posterior reversible encephalitis syndrome $\cdot$ Optic neuropathy $\cdot$ Medication toxicity

\begin{abstract}
Purpose: To report a case of asymmetric bilateral optic neuropathy in a patient receiving tacrolimus for immunosuppression after kidney transplantation with subsequent stroke-like symptoms of posterior reversible encephalopathy syndrome (PRES). Method: Case report. In a 54-year-old Hispanic male receiving tacrolimus after orthotropic kidney transplantation, serial ophthalmologic examinations, laboratory studies, and imaging were performed. Results: The patient had deterioration of vision in the left eye with subclinical optic neuropathy in the right eye, with clinical features resembling ischemic optic neuropathy. Additionally, he developed a change in mental state with weakness of extremities. After the cessation of tacrolimus, the visual loss remained but the other neurologic symptoms resolved. Conclusion: Tacrolimus may be associated with optic neuropathy and PRES at the initial presentation to an ophthalmologist.




\section{Case Reports in Ophthalmology}

\section{Introduction}

The rate of renal transplantations has been steadily increasing in the United States as has survival with the use of immunosuppressive agents like tacrolimus (FK506, Prograf). The primary indication for renal transplantation is end-stage renal disease from diabetes [1], which is a population that we see regularly in the ophthalmologic setting. Given this, it is important to recognize tacrolimus as a source of a potential toxic optic neuropathy and to recognize the systemic features to promptly notify the patient and transplantation team. The toxicity has been described as an optic neuropathy [2] as well as cortical blindness associated with bilateral occipital white matter lesions [3]. Tacrolimus has also been shown to cause biopsyproven demyelination [4]. This immunosuppressive therapy can also cause posterior reversible encephalopathy syndrome (PRES) [5], which is diagnosed by characteristic clinical findings which include headache, changes in mental state, and focal neurological deficits and exclusion of other possible causes. There are also characteristic MRI and CT white matter lesions, but these are not always present. We are unaware of any reports of tacrolimus-induced optic neuropathy and PRES presenting concurrently.

\section{Case Report}

A 54-year-old male underwent a renal transplantation for end-stage renal disease secondary to diabetic nephropathy in May 2014. He had diabetes and hypertension but was otherwise healthy with no history of hyperlipidemia. On August 1, 2014, he noted a sudden onset of painless blurred vision with metamorphopsia and impairment of the left eye superior visual field.

He presented as an emergency patient on August 9, 2014 and stated his vision had progressively worsened. He denied any presence of headache, weakness, numbness, or diplopia. He was on several medications including tacrolimus $6 \mathrm{mg}$ twice a day since his transplantation. He was never on cyclosporine. Ophthalmologic examination revealed best corrected visual acuity (BCVA) of 20/20 in each eye. Confrontational visual fields revealed a superonasal defect in the left eye. Pupils were reactive and symmetric without a relative afferent pupillary defect (RAPD). The patient identified 10 of 10 pseudoisochromatic plates in each eye. Motility, intraocular tensions, and anterior slit-lamp examination were normal. Fundus examination revealed a trace edema with hyperemia and a small inferotemporal optic disc hemorrhage in the right eye, while the left eye revealed a diffuse disc edema with a large inferior disc hemorrhage (Fig. 1). The rest of the posterior examination was notable for moderate nonproliferative diabetic retinopathy and vascular tortuosity bilaterally with macular edema in the left eye tracking from the nerve. There were no retinal infiltrates or vitreous cells. Optical coherence tomography (OCT) of the macula revealed subretinal fluid with peripapillary intraretinal fluid in the left eye (Fig. 2). OCT of the optic nerve and retinal nerve fiber layer (RNFL) revealed a thickened RNFL inferiorly in the right eye and significant diffuse thickening in the left eye. Subsequent visual fields also revealed deficits (Fig. 3). MRI of the brain and orbits with and without contrast did not show any enhancement of the optic nerve or enlargement of the subarachnoid space.

The patient presented to the emergency room on September 21,2014, with additional complaints of memory loss, unsteady gait, and episodes of confusion described by his family. He was not found to be in hypertensive urgency/emergency and his infectious and metabolic workup was negative. The repeat MRI with or without contrast showed no mass or acute 
changes. The patient was evaluated by the ophthalmology service on September 23, 2014. The examination was notable for BCVA of 20/25 in each eye and a RAPD in the left eye, but improved optic disc edema bilaterally. RNFL OCT revealed normal RNFL thickness of the right optic nerve (ON) and thickened but improving RNFL of the left ON. Repeat Humphrey visual field showed a stable superior altitudinal defect in the left eye. Tacrolimus was discontinued on September 23, 2014. An alternate immunosuppressive regiment of mycophenolic acid 720 $\mathrm{mg}$ twice a day and prednisone $10 \mathrm{mg}$ daily was initiated the following day. The symptoms of confusion and weakness began to improve by September 26, 2014 and he returned to baseline mental state by October 1, 2014. Belatacept infusions were initiated as well for rejection prophylaxis and the patient was discharged.

The patient had not had any further deterioration of his vision. As of February 2015, the examination revealed a BCVA of 20/20 with a left RAPD and resolution of the optic nerve edema. Repeat RNFL OCT showed normal thickness in the right eye and inferior thinning with mild superior thickening in the left eye. Repeat visual field testing again showed a stable dense superior altitudinal defect in the left eye.

\section{Discussion/Conclusion}

This patient developed asymmetric optic neuropathy while on systemic tacrolimus therapy and subsequently developed symptoms consistent with PRES which promptly responded to discontinuation of tacrolimus. Although the patient did have risk factors for anterior ischemic optic neuropathy, the bilateral nature and concurrent PRES symptoms which resolved with the cessation of tacrolimus are consistent with tacrolimus-induced optic neuropathy and encephalopathy.

Tacrolimus is known to cause optic neuropathy and many mechanisms have been proposed including direct neurotoxic effect causing axonal swelling or a vascular mechanism [6]. Although optic neuropathy is most commonly seen bilaterally, a significant number of these cases initially present with unilateral or asymmetric symptoms, as seen with our patient. Similarly, optic nerve appearance can vary greatly, likely depending on the time frame between the onset of visual symptoms and the examination [7].

We find this association particularly important in an era of increased transplantation and immunosuppression use. Clinicians, including ophthalmologists, should be aware of this association, as we are responsible for treating diabetics who are the primary recipients of renal transplants and with them this immunosuppressive therapy.

\section{Statement of Ethics}

The authors have no ethical conflicts to disclose.

\section{Disclosure Statement}

The authors have no conflicts of interest to declare. 


\section{Case Reports in Ophthalmology}

\section{Funding Sources}

The authors did not receive any funding.

\section{Author Contributions}

U.B. wrote part of the manuscript and cared for the patient discussed. S.S.K. also wrote part of the manuscript and prepared it for submission. All authors read and approved the final version of the manuscript.

\section{References}

1 Kidney Disease Statistics for the United States. NIH Publication No. 12-3895. June 2012. Available from: https://www.niddk.nih.gov//media/Files/KidneyDisease/KU_Diseases_Stats_508.pdf.

2 Brazis PW, Spivey JR, Bolling JP, Steers JL. A case of bilateral optic neuropathy in a patient on tacrolimus (FK506) therapy after liver transplantation. Am J Ophthalmol. 2000 Apr;129(4):536-8.

3 Shutter LA, Green JP, Newman NJ, Hooks MA, Gordon RD. Cortical blindness and white matter lesions in a patient receiving FK506 after liver transplantation. Neurology. 1993 Nov;43(11):2417-8.

4 Venneti S, Moss HE, Levin MH, Vagefi MR, Brozena SC, Pruitt AA, et al. Asymmetric bilateral demyelinating optic neuropathy from tacrolimus toxicity. J Neurol Sci. 2011 Feb;301(1-2):112-5.

5 Wong R, Beguelin GZ, de Lima M, Giralt SA, Hosing C, Ippoliti C, et al. Tacrolimus-associated posterior reversible encephalopathy syndrome after allogeneic haematopoietic stem cell transplantation. $\mathrm{Br}$ ] Haematol. 2003 Jul;122(1):128-34.

6 Helwig U, Müller M, Hedderich J, Schreiber S. Corticosteroids and immunosuppressive therapy influence the result of QuantiFERON TB Gold testing in inflammatory bowel disease patients. J Crohn's Colitis. 2012 May;6(4):419-24.

7 Rasool N, Boudreault K, Lessell S, Prasad S, Cestari DM. Tacrolimus Optic Neuropathy. J Neuroophthalmol. 2018 Jun;38(2):160-6.
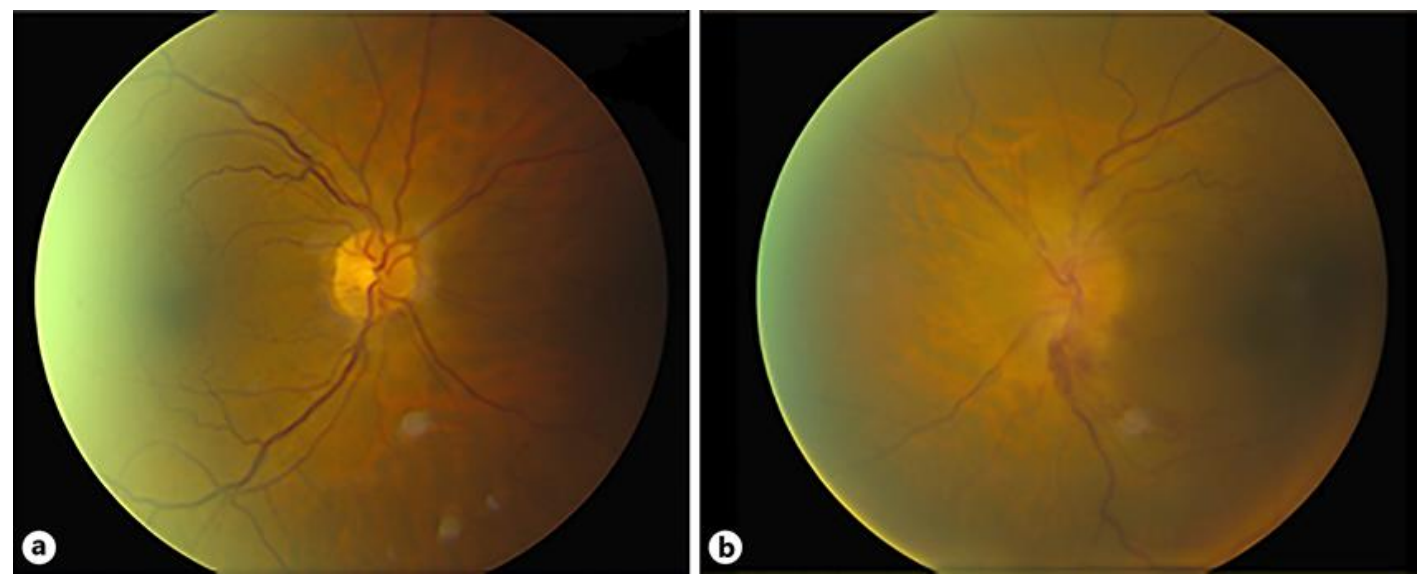

Fig. 1. Fundus photography on August 9, 2014 of the right eye (a) and the left eye (b) with asymmetric optic nerve edema and hemorrhages. 


\section{Case Reports in Ophthalmology}
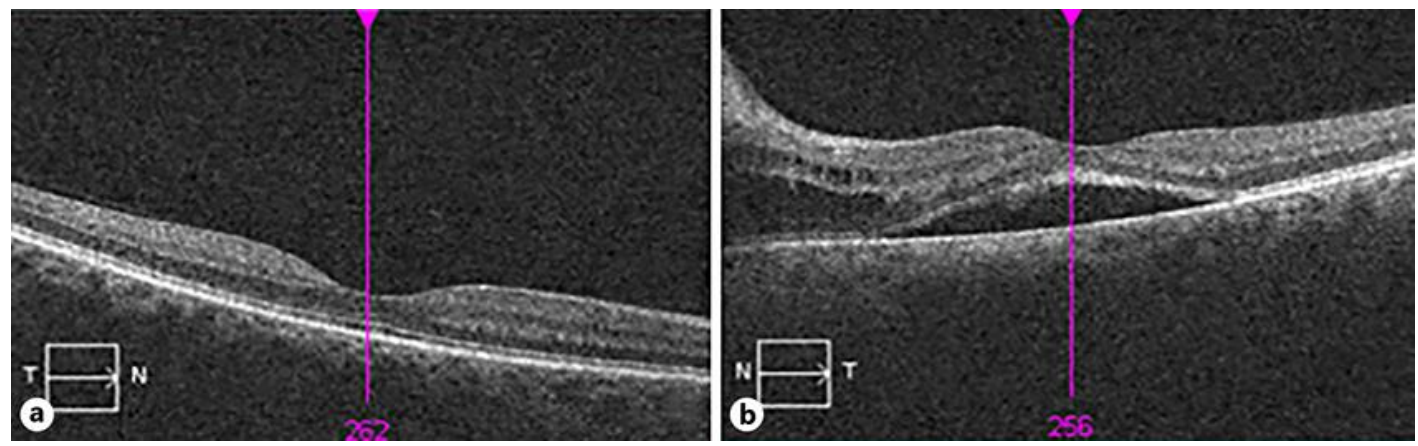

Fig. 2. OCT macula on August 9, 2014, on the right (normal) (a) and left showing intraretinal fluid tracking from the nerve with subretinal fluid (b).
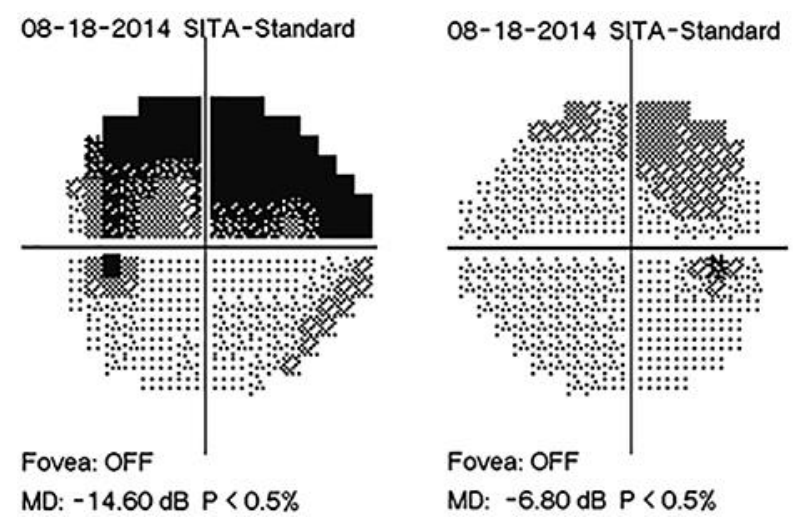

Fig. 3. Humphrey visual field 24-2 obtained on August 18, 2014. The left eye on the left showing a dense superior arcuate defect and the right eye on the right showing a possible superior arcuate defect. 